\title{
Numerical Simulation for Electromagnetic Field Effect of Double-layer Fiber Composites
}

\author{
Baolong Qu \\ College of Chemistry and Environmental Engineering \\ Yangtze University \\ Jingzhou, China \\ e-mail: qubaolong_78@163.com
}

\begin{abstract}
To quantitatively reveal the internal correlation between material microstructure and electromagnetic (EM) field effect by incident EM waves, and to solve the question as to why the numerical simulation research method at present cannot calculate the layered reflectivity and layered absorptivity of double-layer composites, numerical simulations were realized by means of program design and the finite element method. Double-layer fiber composites microstructure is designed. Using the Poynting vector method, the normal reflectivity, transmissivity and absorptivity, the layered reflectivity and layered absorptivity, and the reflectivity for different reflection angles of the double-layer composites are all calculated. The results show that the double-layer fiber composites have a stable EM field effect in a wide frequency range, and the EM energy is most concentrated in the first layer of the double-layer composites. The research method is suitable for any multi-layer composite microstructures.
\end{abstract}

Keywords-composites; electromagnetic field effect; numerical simulation; material microstructure; poynting vector

\section{INTRODUCTION}

The developments of radar detection technology have promoted research into the stealth aspects of aircraft and weapons. There are generally two methods at present: one is to minimize reflections for the detection of electromagnetic (EM) waves by changing the shape of objects; the other is to cover the surface of the target by using a radar absorbing material (RAM) [1,2] or radar absorbing structure (RAS) [3,4] to increase the absorptivity and decrease the reflectivity. In RAS research, double-layer composites are widely used in the aerospace field because they have excellent EM absorption characteristics and can meet the mechanical requirements of engineering components. The double-layer composites can be composed of materials with various functions with optimal structure, which can greatly reduce the reflectivity and improve the absorptivity because they increase the layered multi-reflection and use highly absorbing materials [5]. In experimental research, the EM field effects of double-layer composites which are prepared for the specific experiment are analyzed by a vector network analyzer [6-8]. In recent years, the computer numerical simulation has developed rapidly, which can calculate the EM field effects by accurately simulating the composite structure. The computer numerical simulation becomes a complementary technique to experiment. At present there are a variety of algorithms for the numerical calculation of
EM field effect of composites, such as network ports algorithm based on transmission line [9,10], genetic algorithm [11,12], finite difference time domain method [13], finite volume time domain method [14] and so on. However, traditional research mostly considers the external EM field of composite structures in the EM field effect calculations, and little research has been carried out on the induced EM field of composite structures and the coupling effect between the induced EM field and the external source field. So only the normal reflectivity, transmissivity and absorptivity of whole composites can be calculated, while the oblique reflectivity, the layered reflectivity and the layered absorptivity of double-layer composites cannot be represented and calculated.

In order to overcome the shortcomings of traditional numerical simulation for the EM field effect, this study establishes a finite element model of double-layer composites, which are double-layer carbon fiber composites, and creates an EM field environmental model of the effect analysis. In the computer experiment, EM field effects of the double-layer composite structure are calculated by a new method, that is, the Poynting vector method. Based on the fact that the Poynting vectors can represent the direction and size of EM field energy, they will be projected to the propagation direction and the vertical area with the propagation direction of EM waves, then the components of the Poynting vector in different directions will be used to represent various EM field energies. The numerical simulation calculates normal reflectivity, oblique reflectivity in different angles, transmissivity, absorptivity, layered reflectivity and layered absorptivity of the double-layer composites for plane EM waves. Finally, theoretical analyses are applied to the results of the numerical simulation.

\section{MATHEMATICAL MODEL AND FINITE ELEMENT MODEL}

\section{A. Mathematical model}

The direction and size of EM energy flow can be represented by the Poynting vector. In this article, we use the Poynting vector to characterize the EM field effect.

In the time-harmonic EM field, the instantaneous Poynting vector is

$$
\boldsymbol{P}(\mathrm{t})=\boldsymbol{E}(\mathrm{t}) \times \boldsymbol{H}(\mathrm{t})
$$

Where: $\mathrm{E}(\mathrm{t})$-the instantaneous value of electric field intensity, $\mathrm{H}(\mathrm{t})$-the instantaneous value of magnetic field intensity

In the time-harmonic EM field, the time average value 
of poynting vector is

$$
\boldsymbol{P}_{a v}=\frac{1}{2} \operatorname{Re}\left\{\boldsymbol{E} \times \boldsymbol{H}^{*}\right\}
$$

Where: E- the electric field intensity, $\mathrm{H}^{*}$ - the complex conjugate of the magnetic field intensity.

The time average value of the Poynting vector is independent of time, indicating the complex power flux density. The real part of the Poynting vector is the average power density (active power flux density), units of $\mathrm{W} / \mathrm{m}^{2}$, representing energy flow. The imaginary part of the Poynting vector is the reactive power flow density, units of Var, representing energy exchange. Therefore, in the computer experiment, we should calculate the EM effect using the real part of the Poynting vector.

In the simulation, the Poynting vector is present on each element of the double-layer composite structure. It can be used to calculate reflectivity, transmissivity and absorptivity of EM waves by the direction and size of the Poynting vector.

The value of the real part of the Poynting vector is extracted on each element of the microstructure in the Cartesian coordinate system. These Poynting vectors will be projected to the propagation directions and the vertical area with the propagation direction of EM waves, the projection value representing the flow of EM energy in different directions. Then, they are added as an absolute value, and we get the sum of them all, that is, $\mathrm{P}_{\text {sum }}$ as the total energy value. The angle between the Poynting vector of a microstructural element and the propagation direction of the EM wave can be assumed to be $\alpha$; then, $P_{\text {sum }}$ is calculated for the element of microstructure, that is:

$$
\boldsymbol{P}_{\text {sum }}=\sum(|\boldsymbol{P} * \sin \alpha|+|\boldsymbol{P} * \cos \alpha|)
$$

The component of the Poynting vector that is the projection value in the vertical direction with the propagation direction of EM waves is defined as the absorption energy. The absorptivity of the microstructure can be calculated as:

$$
\mathrm{A}=\frac{\sum(|\boldsymbol{P} * \sin \alpha|)}{\sum(|\boldsymbol{P} * \sin \alpha|+|\boldsymbol{P} * \cos \alpha|)}
$$

The Poynting vector component that is opposite to the propagation direction of EM waves is defined as the reflection energy, and the component in the same direction is defined as the transmission energy. The numerator is replaced as $\sum\left|(\boldsymbol{P} * \boldsymbol{c o s} \alpha)_{-}\right|$and $\sum\left|(\boldsymbol{P} * \boldsymbol{c o s} \alpha)_{+}\right|$, and then we get the reflectivity and transmissivity of the doublelayer composites.

The Poynting vector extracted from different layers of the double-layer composites will be projected to the vertical area with the propagation direction of EM waves. Then, the projection values are added as an absolute value, and we get the sum of them all, that is, the absorption energy in different structural layers of the double-layer composites. Finally, the total energy value is divided by the sum, and we get the layered absorptivity of doublelayer composites, that is:

$$
\mathrm{A}_{\mathrm{i}}=\frac{\sum\left(\left|\boldsymbol{P}_{i} * \sin \alpha\right|\right)}{\sum(|\boldsymbol{P} * \sin \alpha|+|\boldsymbol{P} * \cos \alpha|)}
$$

In which $A_{i}$ is the absorptivity of the ith layer of the structure and $\mathrm{P}_{\mathrm{i}}$ is the element Poynting vector of the ith layer of the structure

We need to calculate the component in the reflection direction of the Poynting vector before calculating the reflectivity in a direction. There is an angle between the reflection direction and the Poynting vector. We just find the cosine of the angle, and the component of the Poynting vector can be calculated in the reflection direction. Then, we get the sum of them, and divide the total energy value by the sum, and we get the reflectivity in the required direction.

The component of the Poynting vector in the reflection direction is calculated. We create a virtual vector to the element coordinate system origin as a starting point in the reflection direction, named as $\mathrm{V}$. In the Cartesian coordinate system, the Poynting vector is $\overrightarrow{\boldsymbol{P}}=\left(\boldsymbol{P}_{\boldsymbol{x}}, \boldsymbol{P}_{\boldsymbol{y}}, \boldsymbol{P}_{z}\right)$, and the reflection direction vector is $\vec{V}=\left(\boldsymbol{V}_{x}, \boldsymbol{V}_{\boldsymbol{y}}, \boldsymbol{V}_{z}\right)$. The angle between the Poynting vector of the element and the reflection direction can be named as $\beta$, shown in Fig. 1 , and then we get the cosine of the angle, that is:

$$
\cos \beta=\frac{\overrightarrow{\boldsymbol{V}} \square \overrightarrow{\boldsymbol{P}}}{|\overrightarrow{\boldsymbol{V}}| \overrightarrow{|\boldsymbol{P}|}}=\frac{\boldsymbol{V}_{x} \boldsymbol{P}_{x}+\boldsymbol{V}_{y} \boldsymbol{P}_{y}+\boldsymbol{V}_{z} \boldsymbol{P}_{z}}{\sqrt{\boldsymbol{V}_{x}^{2}+\boldsymbol{V}_{y}^{2}+\boldsymbol{V}_{z}^{2}} \cdot \sqrt{\boldsymbol{P}_{x}^{2}+\boldsymbol{P}_{y}^{2}+\boldsymbol{P}_{z}^{2}}}
$$

In which $\mathrm{P}_{\mathrm{x}}, \mathrm{P}_{\mathrm{y}}, \mathrm{P}_{\mathrm{z}}$ and $\mathrm{V}_{\mathrm{x}}, \mathrm{V}_{\mathrm{y}}, \mathrm{V}_{\mathrm{z}}$ are the components of the Poynting vector and reflection vector, respectively, in the element coordinate system.

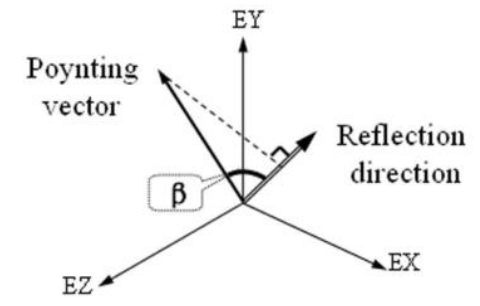

Figure 1. The angle between the Poynting vector and reflection direction in the element coordinate system

The component of the Poynting vector in the reflection direction is $\overrightarrow{\boldsymbol{P}} * \cos \beta$.

$$
\overrightarrow{\boldsymbol{P}} * \cos \beta=\frac{\boldsymbol{V}_{x} \boldsymbol{P}_{x}+\boldsymbol{V}_{y} \boldsymbol{P}_{y}+\boldsymbol{V}_{z} \boldsymbol{P}_{z}}{\sqrt{\boldsymbol{V}_{\boldsymbol{x}}^{2}+\boldsymbol{V}_{y}^{2}+\boldsymbol{V}_{z}^{2}}}
$$

Here, the required value is the reflection energy, so the projection component having a direction the same as the reflection direction can be added to the sum of the reflection energy, and the opposites cannot be included the sum. Then, the reflectivity in any direction is:

$$
\mathrm{R}_{\mathrm{d}}=\frac{\sum\left|(\overrightarrow{\boldsymbol{P}} * \cos \beta)_{+}\right|}{\sum(|\boldsymbol{P} * \sin \alpha|+|\boldsymbol{P} * \cos \alpha|)}
$$

\section{B. The parts of the finite element model}

We established a geometric model and divided it into an effective mesh, shown in Fig. 2. The model included the following four parts.

Double-layer composites: the microstructure model consists of double-layer fiber composites, shown in Fig. 3. 


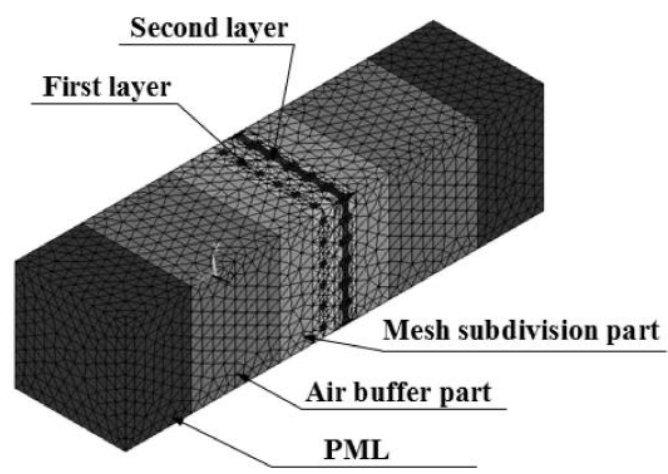

Figure 2. The numerical model of simulation

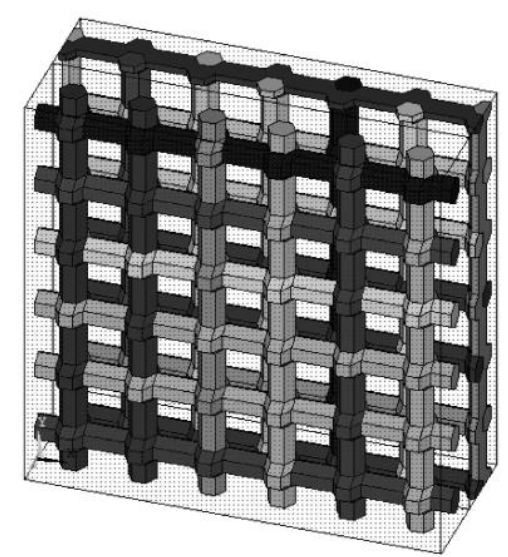

Figure 3. Inner structure of the double-layer composites

Perfectly matched layers (PMLs): also called farfield absorption parts. This is an artificial truncation boundary around the model in order to obtain an accurate simulation. PMLs have good absorption characteristics for the incident EM wave. PMLs can reduce the size of the computational domain effectively with very small numerical reflections, so they can simulate the far-field radiation characteristics of EM waves. Generally, the element layers of PML are not less than six.

The air buffer part: the mesh cannot be directly transited from the microstructure element to the PML element. So there is a four- or five-layer element between them, with the properties of air, called the air buffer part. In addition, the model needs to set up the incident port and the receiving port of EM waves in the middle of this part, and the functions of the ports include loading plane wave and extract data.

The mesh subdivision parts: the main function is the mesh transition from the microstructure to the air buffer part.

\section{Boundary conditions and load}

\section{1) Perfect electric conductor (PEC) boundary conditions}

It is also called electric wall boundary conditions. The tangential component of vector electric field is 0 on the boundary, that is, $n \times \vec{E}=0$. The PEC conditions can be defined by the freedom constraints on the boundary. Generally, PEC boundary conditions are believed the ideal boundary conditions, the losses can be ignored, that is, $\sigma \rightarrow \infty$. In consideration of the PEC boundary conditions, we need not set up the surrounding analyzed microstructure, but just apply an electric field with a tangential component defined as 0 on the boundary surfaces. Analyzed microstructure can be considered as a small part from a relatively infinite object, so the PEC boundary conditions should be applied. In addition, the PEC boundary conditions should be applied on the outside surfaces of the PMLs in order to simulate the far-field absorption effect.

2) Coupling degrees of freedom (DOF) boundary conditions

It is also called cyclic symmetry boundary conditions. It can obtain a correct solution by coupling DOF on the nodes of boundary, and it is suitable for the analysis of periodic symmetry structure and array structure. The sizes of engineering components belong to the macro range. Element numbers are excess imagination if numerical simulations establish detailed microstructure of the component in the FEM analysis, and personal computer can not realize such a large scale calculation. So the representative volume element (RVE) of the component is selected to reflect its macroscopic characteristic for numerical analysis. The representative volume element (RVE) mentioned above refers to the analyzed microstructure. It and its surrounding region have the same structure, materials and properties, so we impose coupling DOF boundary conditions on the model.

3) Plane EM wave load

Generally, the distances from the EM wave source to the detected object are relatively large. So the excitation is considered as a plane EM wave on the detected object. The plane EM wave is loaded by the port excitation. In this study, we apply a cosine wave load, and its propagation direction is along the negative $\mathrm{Z}$-axis.

\section{NUMERICAL SIMULATION AND ANALYSIS OF RESULTS}

\section{A. Numerical simulation}

Single-layer composites often cannot meet the engineering requirements of high absorptivity and low reflectivity. But double-layer composites have wide application in the field of aviation and aerospace, with characteristics of good absorption properties for EM waves, high strength and low density. One can improve EM scattering, interlaminar multiple reflections and heat loss by the design of composite structures and the adjustment of EM parameters[15], and thus achieve improved absorptivity and reduced reflectivity.

The double-layer composite structure goes to make up double-layer fiber composites in this study, as shown in Fig. 3. The matrix phase is polystyrene and the reinforcement phase is carbon fiber. The carbon fiber has a two-dimensional weave structure. Single layer thickness of the fiber composites is $30 \mu \mathrm{m}$, the diameter of carbon fiber being $10 \mu \mathrm{m}$. The sectional shape of carbon fiber is designed as a regular hexagon in order to reduce the number of elements of the numerical simulation. So the total thickness of the composites is $60 \mu \mathrm{m}$. Width and height of the RVE are $150 \mu \mathrm{m}$. The double-layer composites are defined by the first layer and the second layer along the propagation direction of EM waves. 
According to the relevant EM theory and finite element model, using the finite element analysis software ANSYS, we established a numerical model for EM field effect analysis of the double-layer composite microstructure, and meshed effective element, analyzed and calculated the EM field effect of the model based on the Poynting vector method. Element size is defined as $25 \mu \mathrm{m}$. After meshing the model, the total number of elements is 70272 , and the total number of nodes is 98 996. Each node has one DOF, that is, magnetic vector, so general DOF is 98996 in the finite element model. The meshed result is shown in Fig. 1

\section{B. Results and discussion}

The plane EM wave is loaded along the negative $\mathrm{Z}$ axis propagation. Normal reflectivity, absorptivity and transmissivity of the double-layer composites, layered absorptivity and layered reflectivity of the fiber composite layers, and the different reflectivity in the directions with angles $0^{\circ}, 30^{\circ}, 45^{\circ}$ and $60^{\circ}$ in the opposite direction to EM wave propagation are all calculated.

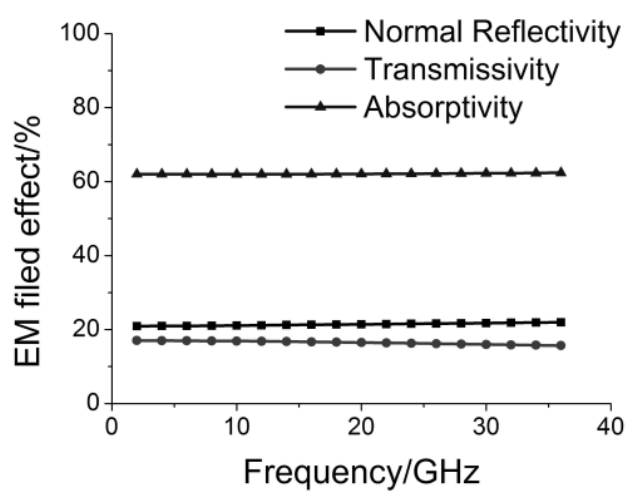

Figure 4. EM field effect of double-layer composites

The double-layer composites have stable reflectivity, transmissivity and absorptivity for the incident plane EM waves in the range of frequency from 2 to $36 \mathrm{GHz}$. The absorptivity is more than $60 \%$, and the reflectivity is relatively low, less than $20 \%$. The results are shown in Fig. 4. There are two reasons for the high absorptivity. Firstly, the EM field produces multiple reflections in two fiber composite layers. Because of the EM loss of the composites, the EM field has a greater attenuation for short distances, which is the main factor for the high absorptivity of the double-layer composites. Secondly, the carbon fibers are connected to each other, and have high EM loss. The induced current can flow freely in the double-layer composites, and it can form an annular flow which is similar to the eddy current effect. The annular currents mean that most of the EM energy is bounded within the double-layer composites, which is one of the reasons for the high absorptivity. The reflectivity is relatively low, the main reason being that the inner structure of the doublelayer composites is periodic and symmetrically distributed, and the induced EM fields of the various parts can be counteracted to a great extent.

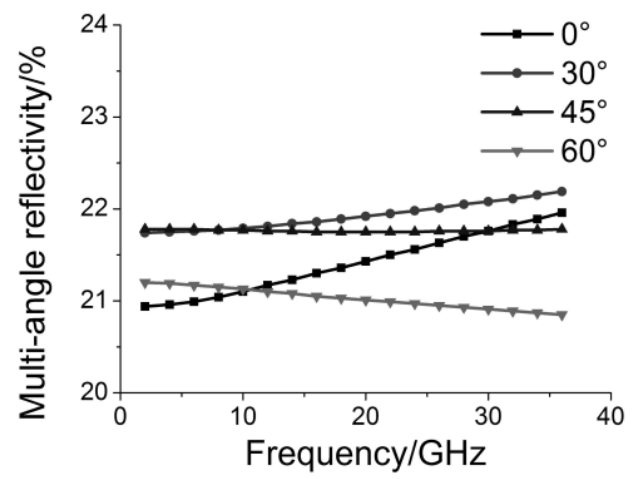

Figure 5. Reflectivity in different directions

The reflectivity curves of the double-layer composites for the normal incident EM wave in different reflection directions are shown in Fig. 5. The reflection angle is defined as the angle between the reflection direction and the opposite direction of the EM wave. It can be seen from the curves that the reflection angle $0^{\circ}$ is the direction of the EM wave source, so $0^{\circ}$ reflectivity is equivalent to normal reflectivity, and the reflectivity in other directions is equivalent to oblique reflectivity. In the range of frequency from 2 to $36 \mathrm{GHz}$, the reflectivity for different angles has different trends. With an increase of frequency, the reflectivity for $0^{\circ}$ and $30^{\circ}$ increases gradually, that for $45^{\circ}$ is stable, but that for $60^{\circ}$ decreases gradually. At lower frequency, the reflectivity for $0^{\circ}$ and $30^{\circ}$ is higher, while at higher frequency, the reflectivity for $60^{\circ}$ is lower. These results concern the EM parameters of carbon fiber and the reflection angle.

The layered absorptivity of the double-layer composites is shown in Fig. 6. The first layer has a significant contribution: the layered absorptivity is about $86 \%$ of the total absorptivity. The second layer has a low layered absorptivity, about $14 \%$ of the total absorptivity. The layered reflectivity of the double-layer composites is shown in Fig. 7. The situation is similar to that of the absorptivity curve. The layered reflectivity of the first layer has a significant contribution, about $84 \%$ of the total reflectivity. The layered reflectivity of the second layer has a low contribution, about $16 \%$ of the total reflectivity. The main reason for these results is that most of the EM energy is concentrated in the first layer as a result of the skin effect, so the absorptivity and reflectivity of the first layer are higher than those of the second layer.

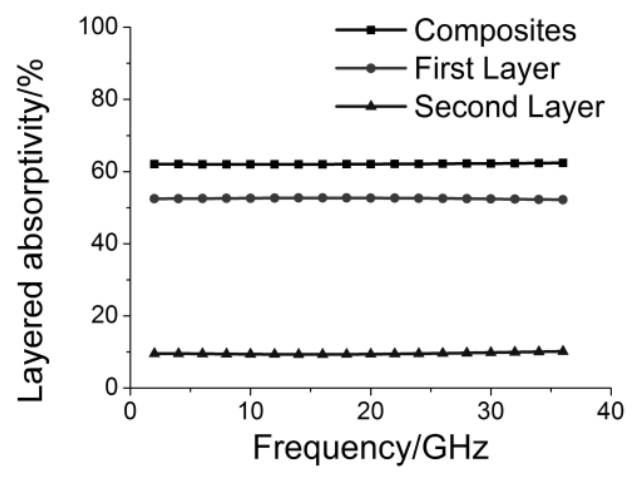

Figure 6. Layered absorptivity of double-layer composites 


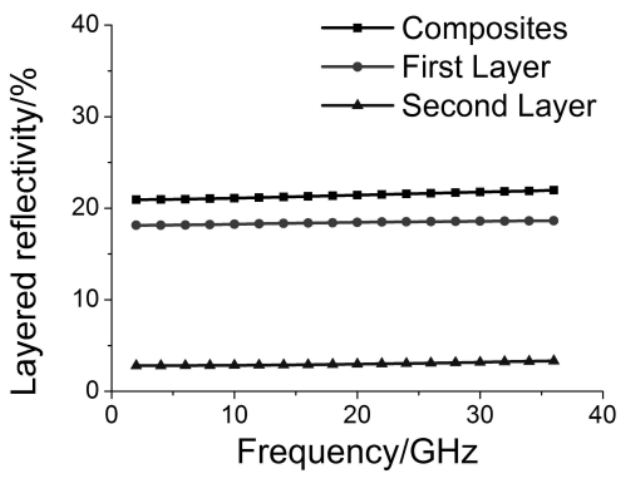

Figure 7. Layered reflectivity of double-layer composites

In addition, the two-dimensional braided carbon fibers form a complex network structure. The network structure can be regarded as different sized and interconnected grids which are composed of the minimum grid hole as the basic unit. The grids will produce the induced EM fields which correspond to the excitation EM fields of different frequency, so the reflectivity, transmissivity and absorptivity of the double-layer composites are relatively stable for different frequencies.

\section{CONCLUSIONS}

The finite element model of double-layer composites, which are composed of carbon fiber and polystyrene, is established based on engineering applications. The sectional shape of the carbon fibers is simplified appropriately in the model. The EM environment model was established, and the EM field effect of the doublelayer composites was calculated and analyzed. The normal reflectivity, transmissivity and absorptivity of the doublelayer composites are calculated by the Poynting vector decomposition in different directions. The layered reflectivity, layered absorptivity and oblique reflectivity for different angles of the double-layer composites are calculated, and the reflectivities of different reflection angles are also calculated. Theoretical analyses of the results show that the numerical simulation is viable and valid. The results show that: (1) EM field effects of the double-layer composites are very stable in the frequency range from 2 to $36 \mathrm{GHz}$, (2) the reflectivity of different angles shows different trends and (3) the EM energy is most concentrated in the first layer of the double-layer composites. The research method provides new ideas for the calculation of layered EM field effects of double-layer composites and the reflectivity for different reflection angles.

\section{ACKNOWLEDGMENT}

Author acknowledge the financial support provided by the National Natural Science Foundation of China (No. 50271016, 50571042).

\section{REFERENCES}

[1] Zhang $\mathrm{H}$, Zhang J, Zhang H. Computation of radar absorbing silicon carbide foams and their silica matrix composites[J]. Computational Materials Science, 2007, 38(4): 857-864.

[2] Nicolaescu I. Radar absorbing materials used for target camouflage $[\mathrm{J}]$. Journal of Optoelectronics and Advanced Materials, 2006, 8(1): 333-338.

[3] Fan H L, Yang W, Chao Z M. Microwave absorbing composite lattice grids[J]. Composites Science and Technology, 2007, 67(15): 3472-3479.

[4] Chin W S. Development of the composite RAS (radar absorbing structure) for the $\mathrm{X}$-band frequency range $[\mathrm{J}]$. Composite Structures, 2007, 77(4): 457-465.

[5] Raj C D, Rao G S, Jayasree P V Y, et al. Analysis of reflectivity and shielding effectiveness of absorbing material-conductor laminate for electromagnetic compatibility $[\mathrm{J}]$. Journal of Electromagnetic Analysis and Applications, 2010,(2): 318-323.

[6] Zou T, Shi C, Zhao N. Microwave absorbing properties of activated carbon-fiber felt dipole array/epoxy resin composites[J]. Journal of Materials Science, 2007, 42(13): 4870-4876.

[7] Wu X, Feng Y, Liu Y, et al. Preparation and microwave absorption properties of barium ferrite powder coated with $\mathrm{Ni}$ Co-P alloy coating by electroless plating $[\mathrm{J}]$. Journal of the Chinese Ceramic Society, 2009, 37(2): 311-316.

[8] Kim J B, Kim C G. Design of broad band radar absorbing composite laminates[C]. Advanced Materials Research. 2010, 123 : 951-954.

[9] Kim H T, Liu K, Lee Y T, et al. Structure-dependent dielectric constant in thin laminate substrates[C]. Electronic Components and Technology Conference (ECTC), IEEE, 2011: 1717-1722

[10] Kim J W, Kim S S. Microwave absorbers of two-layer composites laminate for wide oblique incidence angles[J]. Materials \& Design, 2010, 31(3): 1547-1552.

[11] Zhang Z Q, Wang L G, Wang E Z. Microwave absorbing properties of radar absorbing structure composites filling with carbon nanotubes[C]. Advanced Materials Research. 2011, 328: 1109-1112.

[12] Matouš K, Dvorak G J. Optimization of electromagnetic absorption in laminated composite plates[J]. IEEE Transactions on Magnetics, 2003, 39(3): 1827-1835.

[13] Schultz J W, Cieszynski B. Sub-wavelength measurement of electromagnetic inhomogeneities in materials $[\mathrm{J}]$. Antennas and Propagation Magazine, 2007, 49(4): 225-230.

[14] Fumeaux C, Baumann D, Almpanis G, et al. Finite-volume timedomain method for electromagnetic modelling: strengths, limitations and challenges[J]. International Journal of Microwave and Optical Technology, 2008, 3(3): 318-328.

[15] Li H, Wang J, Huang Y, et al. Microwave absorption properties of carbon nanotubes and tetrapod-shaped $\mathrm{ZnO}$ nanostructures composites $[\mathrm{J}]$. Materials Science and Engineering: B, 2010, 175(1): 81-85. 